18

\title{
Photoluminescence Properties of Thin-Film Nanohybrid Material Based on Quantum Dots and Gold Nanorods*
}

\author{
(C) S.A. Goncharov ${ }^{1}$, V.A. Krivenkov ${ }^{1}$, P.S. Samokhvalov ${ }^{1}$, I. Nabiev $^{1,2}$, and Y.P. Rakovich ${ }^{1,3,4,5}$ ब \\ ${ }^{1}$ National Research Nuclear University MEPHI (Moscow Engineering Physics Institute), \\ 115409 Moscow, Russia \\ ${ }^{2}$ Laboratoire de Recherche en Nanosciences, LRN-EA4682, Université de Reims Champagne-Ardenne, \\ 51100 Reims, France \\ ${ }^{3}$ Donostia International Physics Center (DIPC), \\ 20018 Donostia-San Sebastiaín, Spain \\ ${ }^{4}$ Centro de Física de Materiales (MPC, CSIC-UPV/EHU), \\ 20018 Donostia-San Sebastian, Spain \\ ${ }^{5}$ IKERBASQUE, Basque Foundation for Science, \\ 48013 Bilbao, Spain \\ e-mail: goncharov.semyon@gmail.com, yury.rakovich@ehu.es
}

Received July 6, 2018

Semiconductor quantum dots (QDs) have been demonstrated to be a promising material for developing innovative optoelectronic systems and lasers. The strong and weak coupling effects between localized plasmons in noble metal nanoparticles and excitons in QDs can modulate photoluminescence properties of the latter, scaling up their applications. In particular, these effects can strongly affect the photoluminescence (PL) lifetime of QDs, opening prospects for significantly increasing the quantum yield of the biexciton emission in single QD. Here, we provide a convincing proof of the formation of many-exciton states in hybrid material based on CdSe/ZnS/CdS/ZnS core/multishell QDs and gold nanorods (NRs) embedded in thin films of PMMA. The presence of NRs causes at least an order-of-magnitude decrease in the PL lifetimes of single QD. The obtained results have demonstrated the possibility of detecting biexciton states in QDs as the main component of emission of the hybrid QD-NR material.

DOI: $10.21883 /$ OS.2018.11.46833.216-18

\footnotetext{
* International Conference „PCNSPA 2018 - Photonic Colloidal Nanostructures: Synthesis, Properties, and Applications", Saint Petersburg, Russia, June 4-8, 2018.

Полный текст статьи опубликован в английской версии журнала.
} 